\title{
Peroxisome Proliferator-Activated Receptors - Alpha in Chronic Inflammation - Mini-Review
}

\author{
Elena Popa ${ }^{1 *}$, Florin Zugun-Eloae ${ }^{2,3}$, Mihaela Zlei ${ }^{3}$, Maria Traian ${ }^{1}$, \\ Agnes Bacusca ${ }^{1}$, Andrei Emilian Popa ${ }^{4}$, Adorata Elena Coman ${ }^{1}$ \\ ${ }^{1}$ Department of Family Medicine, Faculty of Medicine, „Grigore T. Popa” University of Medicine \\ and Pharmacy, lasi, Romania \\ 2Department of Immunology, Faculty of Medicine, „Gr T Popa” University of Medicine and \\ Pharmacy, lasi, Romania \\ 3Laboratory of Molecular Biology, Regional Institute of Oncology, Iasi, Romania \\ ${ }^{4}$ Faculty of Medicine, „Grigore T. Popa” University of Medicine and Pharmacy, lasi, Romania \\ *elenapopadr@yahoo.com
}

Keywords: Chronic Inflammation; PPARa; Metabolic Syndrome

\begin{abstract}
The pathogeny of the metabolic syndrome (MetS) is not fully elucidated, but a link between visceral obesity and the increase of the proinflammatory response was proven. Atherosclerosis, perceived as a metabolic complication, draws attention to the peroxisome proliferator-activated receptors- alpha (PPAR $\alpha$ ). PPAR $\alpha$ receptors are transcription factors involved in lipid metabolism, inflammation and atheromatosis. Hence, it interferes in the pathogeny of cardiovascular diseases and other chronic diseases too (neurological, psychical, neoplasical). The study of the expression of PPAR $\alpha$ and its modulation on different level may be beneficial in the treatment of metabolic syndrome, intervening in the modulation of another proinflammatory factors.
\end{abstract}

\section{Introduction}

Many chronic diseases have been associated with a persistent, reduced level of proinflammatory factors. The discovery of insulin resistance and of type 2 diabetes is closely related to the infiltration of immune cells and chronic inflammation in the adipose tissue. The activated immune cells and chronic inflammation play a major role in cardiovascular diseases, especially in the pathogenesis of atherosclerosis $[1,2]$. The peroxisome proliferator-activated receptors - PPARs - are part of the category of nuclear receptors of the protein type, similar to receptors for steroid or thyroid hormones [3-5]. Independent groups of researchers noticed in the sixth decade of the twentieth century an increase in the number of hepatic peroxisomes after administering lipid-lowering drugs (clofibrate). An increase in fatty acid oxidation in hepatic peroxisomes, as well as an increased risk of carcinogenesis in the long-term administration of these drugs was simultaneously observed [5-7]. Later, in the 90s, the nuclear receptor called PPAR (peroxisome proliferator-activated receptor) was identified and cloned in mice and later in humans and amphibians $[8,9]$.

\section{PPAR - Mechanism of Action}

The peroxisome proliferator-activated receptors (PPAR), which comprise three PPAR isoform: PPAR $\alpha, \operatorname{PPAR} \gamma$ and PPAR $\delta$ (10), act as transcription factors, belonging to the nuclear receptor superfamily [4-6], The mechanism of action of PPAR $\alpha$ (Fig. 1) is similar to that of other nuclear receptors (thyroid or that of vitamin D) [7-9, 11]. The activation of the PPAR receptor determines a change in the structure of the receptor complex, followed by changes in the expression of coded genes. PPAR acts as a ligand-activated transcription factor [7, 12]. 


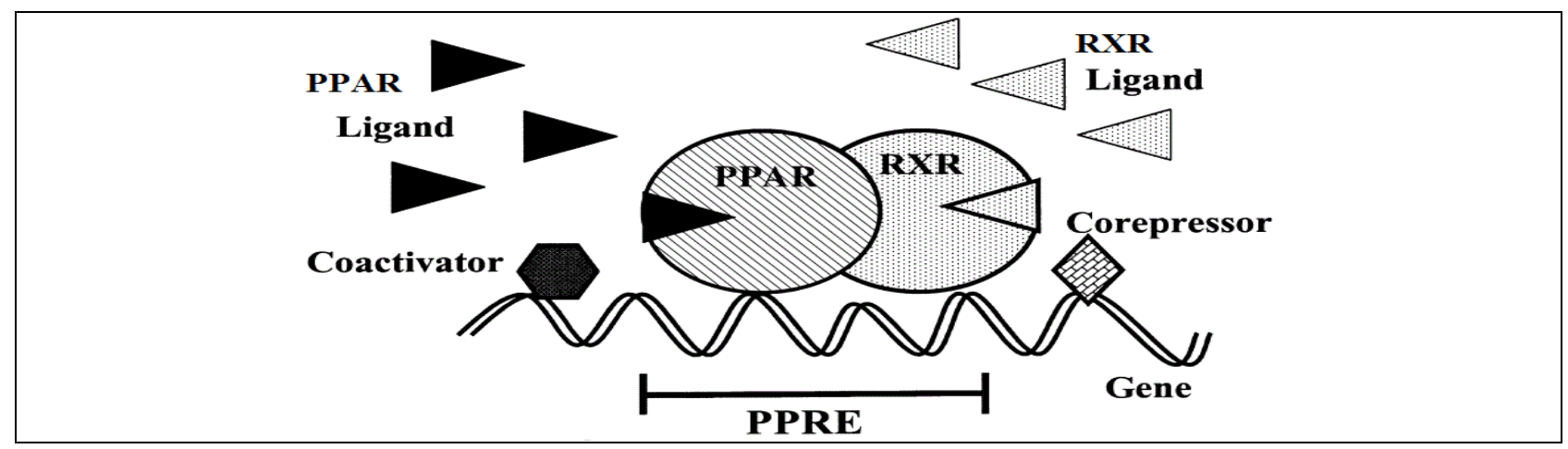

Figure 1. PPAR - mechanism of action - adaptet from Roberts-Thompson S J [7].

After stimulation by the ligands ("Peroxisome Proliferator-PPs") it unites with the Retinoid Receptor X (RXR), forms a heterodimer and binds to the specific "PPAR response elements (PPREs)" in the promoter of the target genes $[7,8,12]$.

\section{PPAR Alpha in Chronic Inflammations and Diseases}

Peroxisome Proliferator-Activated Receptors-Alpha (PPAR $\alpha$ ) have a significant role in the regulation of lipids and lipoproteins metabolism, chronic inflammation and atherogenesis [14]. PPAR $\alpha$ can be activated by endogenous ligands (fatty acids) and pharmacological agents (such fibrates) (Table 1) [10].

Table 1. PPAR $\alpha$ ligands.

\begin{tabular}{|l|l|}
\hline Endogenous & Synthetic \\
\hline Unsaturated fatty acids & Fibrates (fenofibrate, bezafibrate, clofibrate) \\
Saturated fatty acids & WY - 14643 \\
VLDL & Etilenoxide \\
Leucotriene B4 & Ftalates \\
8- Hydroxyeicotetraenoic acid & Perfluorooctanesulfonic acid \\
\hline
\end{tabular}

Experimental studies [15-17] have shown that the degradation by oxidation of proinflammatory molecules such as LTB4 (ligand of PPAR $\alpha$ ) or arachidonic acid is enhanced after PPAR $\alpha$ stimulation. In animal models, in gene-modified mice PPAR $\alpha^{-/-}$, it was detected a prolonged inflammatory response and these data are related to lack of degradations of chemotactic inflammatory eicosanoids LTB4 $[16,17]$. Furthermore, in wild-type mice, but not in PPAR $\alpha^{-/-m i c e, ~ t r e a t m e n t ~ w i t h ~ W Y-14643, ~}$ a ligand of PPAR $\alpha$, suppressed a number of acute phase genes such as fibrinogen, serum amyloid Pcomponent, lipocalin 2, serum amyloid A-2, metallothioneins [15, 17].

In human cells, it was shown that activators of PPAR $\alpha$ inhibit the expression of vascular cell adhesion molecule-1 (VCAM-1) gene in endothelial cells, while. In human aortic muscle cells, the nuclear receptors decrease interleukin-1 (IL-1) and induce the production of interleukine-6 (IL-6), prostaglandins and the expression of cyclooxygenase-2 (COX-2) gene [17, 20]. In vivo, in patients with dyslipidemia and metabolic syndrome, treatment with fibrates decreases the circulating level of systemic inflammations markers, such as high-sensitivity C-reactive protein (hsCRP), IL-6 [15, 17, 19]. This effect is independent of lipoprotein metabolism and does not change insulin sensitivity. 


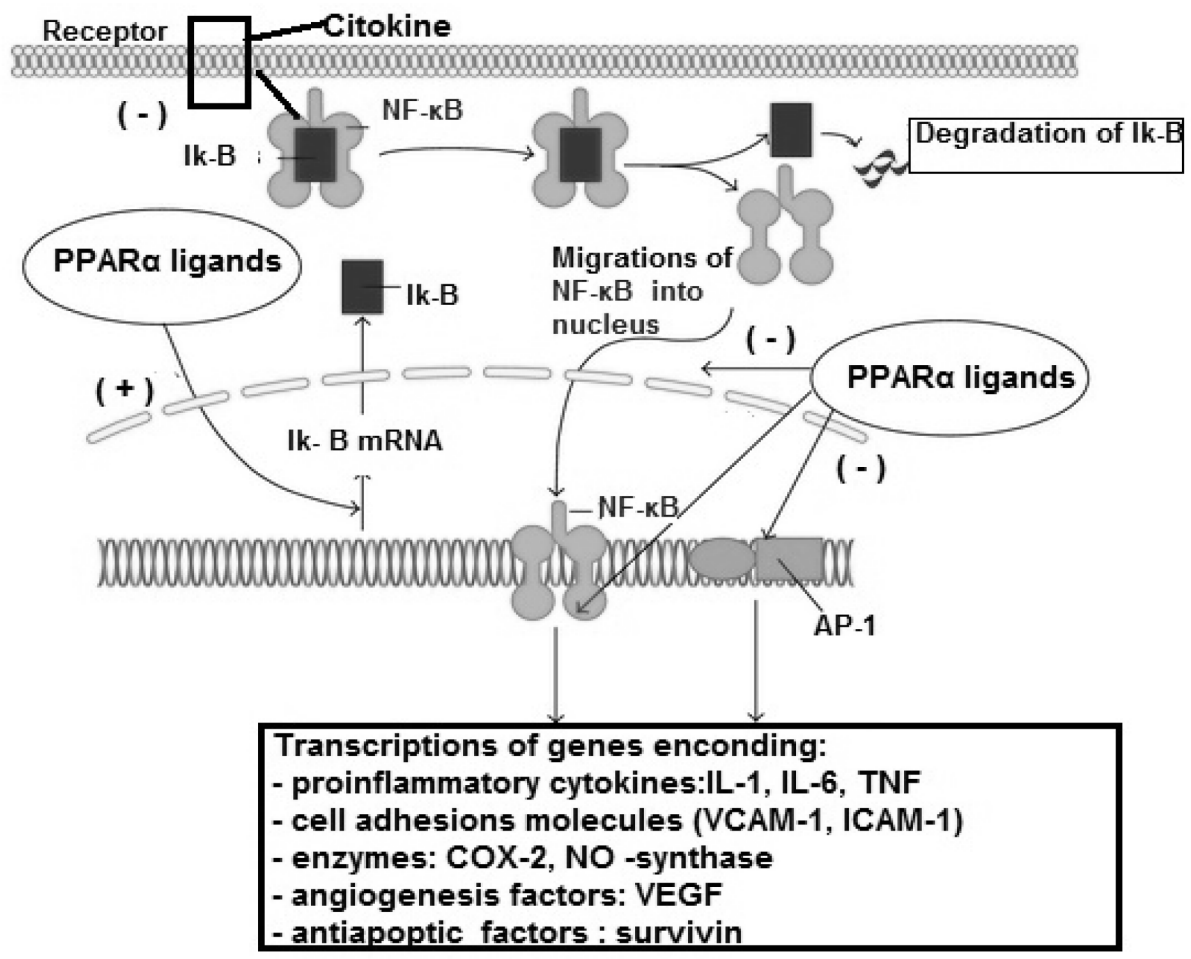

Figure 2. The anti-inflammatory effects of PPAR ligands - adapted from Shirinsky (2011) is mediated via $\mathrm{NF}-\kappa \mathrm{B}$ through following mechanisms $[15,17]$ :

- downregulation of the expression of signal transducing receptor components

- induction of Ik-B expression

- inhibition of nuclear factor $\mathrm{kB}$ into nucleus

- decreasing of transcription factor expression levels

- interference with activation of the transcription initiation complex via cofactor interaction.

Recent studies $[15,17,20]$. have shown that the activation of PPAR $\alpha$ is folowed by the decrease of systemic inflammation by antagonising the Nuclear Factor kB (NF-kB) and the transcriptional activities of protein AP-1, leading to reduction on synthesis of proinflammatory molecules (Fig. 2).

The Implications of the Relation between Lipoprotein Metabolism and the Activation of PPAR $\alpha$ in vivo in Inflammation and Atherosclerosis

Many studies have so far established the close relation between free fatty acids and PPAR $\alpha$. There is, however, relatively little information on the manner in which lipoprotein changes could be related to PPAR $\alpha$ response at cellular level. Interestingly, PPAR $\alpha$ actions have been defined using synthetic PPAR $\alpha$ agonists. Lipoprotein lipase (LPL), a member of the triacylglycerol lipase family, is an important enzyme in the hydrolysis of triglyceride-rich lipoproteins (VLDL) with the release of high free fatty acid levels. The activity of this enzyme is intense both in the muscle, where it helps to provides free fatty acids for beta-oxidation and energy generation, as well as in the adipose tissue, where the free fatty acids can be stored as reserves. Recent works have shown that the hydrolysis of VLDL can activate PPARa. In macrophage, LPL activation is done by PPAR $\delta$ (9), while LPL action is mediated by PPAR $\alpha$ in the endothelial cell $[13,19]$. Not all the lipases which liberate free fatty acids act on PPAR $\alpha$, just LPL. A complex made of LPL, lipoproteins and their lipolytic products is described to influence PPAR $\alpha$ responses. 


\section{Relation PPARa - Endothelial Reactivity - Atherosclerosis}

The endothelial lesion determines endothelial dysfunction, manifested by altering the role of the highly selective barrier; it alters antithrombotic properties and favors pro-coagulant properties, by affecting the synthesis and secretion of vasoactive substances, growth factors (mitogens), and lipolytic enzymes. PPAR $\alpha$ are active in the human endothelial cell. Moreover, the PPAR activators are involved in the modulation of the endothelial function. For example, PPAR $\alpha$ activators prevent the recruitment and adhesion of leukocytes at the level of the vascular endothelium by lowering VCAM-1 (vascular cell adhesion molecule), ICAM-1 (intercellular adhesion molecule) and decreasing the E-selectin expression. PPAR $\alpha$ activators regulate the adhesion molecules due to the inhibition of NF-kB $(13,21)$. The endothelial dysfunction includes alterations in any of the functional roles in vivo, including the maintenance of normal vascular tone, the limitation of thrombo-genesis and the protection against leukocytes adhesion $[12,20]$.

\section{Relation PPAR $\alpha$ - Cardiovascular Diseases}

The energy substrates, such as free fatty acids (FFA) and glucose, are involved in generating the energy used for normal cardiac activity. With normal conditions, the ATP is generated by the mitochondrial oxidation of fatty acids (FAO) and glucose, only $2 \%$ or less being derived from anaerobic glycolysis [22-24].

PPAR $\alpha$ are a major transcriptional factor of FFA metabolism in the heart $[24,25]$. The activation of PPAR $\alpha$ in cardiac muscle by FFA, derivate from triglycerides, regulates genes that encode proteins implicated in FFA and glucose metabolism (Table 2) [25, 26].

Table 2. PPAR $\alpha$ target genes in cardiac muscle -adapted from Lopaschuk [25].

$\uparrow$ FFA uptake (i.e Fatty acids translocate/ cluster of differentiation)
$\uparrow$ FFA storage (i.e.Diacylglycerol acyl transferaze - DGAT)
$\uparrow$ FFA $\beta$-oxidation (i.e.carnitine palmitoyl transferase - CPT1, medium
chain acyl-CoA dehydrogenase - MCAD)
$\downarrow$ glucose oxidation (ie: pyruvat dehydro-genase Kinase 4 - PDK4)

Experimental studies on mice have shown that the overexpression of PPAR $\alpha$ in cardiomyocytes increases the uptake of FFA and beta-oxidation and decreases the glucose oxidation and cardiac lipids accumulation as a consequence of increasing the expressions of genes that are modulated by these nuclear receptors. On the contrary, the deletion of PPAR $\alpha$ gene (PPAR $\alpha-/-$ null mice), determines decrease of myocardial FFA metabolism, increase of glucose oxidation and hepatic lipid accumulation $[25,26]$.

The increased expression of PPAR $\alpha$ receptors in myocardium reproduces the metabolic profile in diabetes: increased myocardial fatty acid oxidation, increased rate of $\beta$-oxidation and decreased glucose levels [22-24]. In chronic diabetes and hypertensive hearts, studies using proteomics [27] have revealed alterations in expression of hypertrophic, metabolic and apoptotic proteins. In experimental model of chronic diabetes and hypertensive in rats, treatment with PPAR $\alpha$ agonists was followed by an attenuation of cell hypertrophy induced by high-glucose- and angiotensin-II levels. Furthermore, agonists of PPAR $\alpha$ can be used as anti-hypertrophic agents for patients with hypertension, in prevention of left ventricular hypertrophy, and beyond in prevention of cardiac remodeling [26, 27].

Many studies have shown the decrease of PPAR $\alpha$ expression after exposure to high blood pressure which stimulates cardiac hypertrophy. The activation of PPAR $\alpha$ in hypertensive animal model improves left ventricular hypertrophy and cardiac remodeling. Study of spontaneously hypertensive rats (SHR) has revealed that the treatment with fenofibrate delay ventricular hypertrophy, fibrosis, and decrease oxidative stress in young SHR with cardiac hypertrophy [28]. These effects of PPAR $\alpha$ stimulation are linked to interactions of PPAR $\alpha$ via proinflammatory pathways (NF kB, AP-1) (Fig. 2). On the contrary, fenofibrate enhances hypertrophy, fibrosis, and oxidative stress in old SHR with cardiac hypertrophy and decreased fatty acids oxidations [28, 29]. 
In a rat myocardial infarction (MI) model of heart failure, downregulation of PPAR $\alpha$ is the initial response after MI (first 4 weeks) [26, 30]. After 6 weeks, PPAR $\alpha$ expression increases and it returns to normal value 20 weeks after MI [31]. This suggests an overload in post MI metabolic state in other cardiac cells, including fibroblasts [26].

PPAR $\alpha$ expression has anti-inflammatory effects and prevents oxidative damage and treatment with PPAR $\alpha$ agonists may inhibit the inflammation in many cells types in chronic cardiovascular diseases [32].

\section{The Interaction between PPAR $\alpha$ and Inflammation in Skeletal Muscles}

The activation of PPAR $\alpha$ receptors at the level of the skeletal muscles is followed by an increase in lipid oxidation and decrease in triglyceride accumulation [20]. The side effects of fibrates on muscle fibers, including myopathy and rhabdomyolysis, have only been observed in rare cases $(<1 \%)$. The mechanism by which these side effects occur is not very clear, the oxidative stress and the tissue alteration induced by the increase in oxidation in the peroxisomes and mitochondria being held responsible [24,33]. The systemic inflammation, the immune system and physical activity exist in an interesting and complex correlation. Moderate regular physical exercise reduces systemic inflammation $[34,35]$. The mechanism by which the PPAR $\alpha$ agonists determine the occurrence of myopathy is not well documented as of yet, but it appears to be the consequence of oxidative stress. To conclude, the modulation and the return to the normal level of the secretion of muscle-derived cytokines is necessary due to the fact that the high serum levels of myokines are related to the discovery of conditions associated with systemic inflammation, including cancer and other conditions entailed by aging (degenerative nervous disorders, depression, sarcopenia) [35, 36].

\section{The Role of PPARa in Kidney Diseases}

The activity of PPAR $\alpha$ is manifested in the organs with a high level of mitochondrial oxidation: liver, renal cortex, heart, and bowel. PPAR $\alpha$ are numerous in the kidney, at the level of the proximal tubule and in the glomerular mesangial cells [37]. Due to the increased expression in kidney proximal tubules, PPAR $\alpha$ are involved in maintaining energy balance in the kidney [38]. PPAR $\alpha$ are involved in oxidative stress, inflammation, blood pressure regulation and renin-angiotensinaldosterone system regulation, therefore influencing the pathogenesis and progression of diabetic nephropathy by indirect route, through their effects on serum glucose level and on the lipid metabolism, and by direct route, through their action on the kidney. These findings suggest that PPAR $\alpha$ is a therapeutic target in treating kidney complications from diabetes. The occurrence of glomerular lesions in PPAR $\alpha$ deficiency is followed by an increase in the production of type IV collagen as well as in the expression of transforming growth factor beta-1 (TGF- $\beta 1$ ) cytokines at this level, suggesting the fact that the activation of PPAR $\alpha$ by its agonists could prevent the expansion of the glomerular matrix and thus the infiltration with the inflammatory cells of the renal glomerulus. The corroboration of many experimental studies resulted in the fact that PPAR $\alpha$ influences inflammatory conditions involving neutrophils and macrophages [38]. In diabetic patients, hyperglycemia, dyslipidemia, endothelial dysfunction, lipotoxicity as well as arterial hypertension contribute to the onset of systemic or local complications, by the activation of pro-inflammatory factors and of renin-angiotensin (RAS), the increase of oxidative stress and of cell apoptosis and the onset of vasculopathies. Furthermore, the onset and development of complications can be attenuated by PPAR $\alpha$ activation [38].

\section{The Relation between PPAR $\alpha$ and Nervous System Disorders}

The idea that Alzheimer's disease is a form of the diabetic disease has gained scientific authority almost ten years ago. Many specialists are now entitled to claim that Alzheimer's disease is a type 3 diabetes mellitus [39]. Placing Alzheimer's disease in the category of type 3 diabetes mellitus should not come as a surprise for healthcare professionals, due to the fact that insulin does not only 
signal the uptake of glucose in the somatic cells of the body, but also regulates brain glucose uptake. This is very important due to the fact that glucose is known as being the primary energy molecule of the brain, representing the only energy substrate for the brain [40]. Currently, it is well know that, at the level of the central nervous system, the brain in itself produces a certain quantity of insulin, and that various parts of the brain are rich in insulin receptors. Moreover, the fact that the cognitive decline is correlated with obesity and metabolic abnormalities which involve insulin is also well established [40].

The role of PPAR in modulating lipid and carbohydrate metabolism is well established [41]. More recently, the importance of PPAR in modulating inflammation has been proven. For example, PPAR $\alpha$ agonists inhibit the production of pro-inflammatory molecules in the peripheral immune cells as well as in the glial cells of the nervous system. Moreover, PPAR $\alpha$ agonists have proven to be effective in suppressing inflammation in the central nervous system in animal models, being useful in treating neurodegenerative diseases [42]. The degenerative disorders of the nervous system are related to the local pro-inflammatory response (neuro-inflammation). The tumor necrosis factor $(\mathrm{TNF} \alpha)$ stimulates the apoptosis of dopaminergic neurons in the substantia nigra, neurons involved in the pathology of Parkinson's disease. The same inflammation-mediated degenerative model, in the context of dysglycemia, is also met in diabetic neuropathy. The pro-inflammatory condition is characterized by the activation of macrophages and monocytes in the lesion caused by various factors. Moreover, an accumulation of pro-inflammatory mediators is produced: TNF- $\alpha$, IL-6, IL- $1 \beta$, alongside COX, generating a pro-inflammatory cascade. PPAR $\alpha$ and fenofibrates reduce pain and inflammation by inhibiting the NF-kB nuclear factor, followed by the reduction of pro-inflammatory mediators, as well as by the reduced activity of certain enzymes which stimulate angiogenesis (iNOS, chymase and metalloproteinase MMP-9) [43].

\section{The Role of PPARa Receptors in Diabetic Retinopathy}

In type 2 diabetes, the FIELD study shows a reduced need for laser treatment in diabetic retinopathy and a possible diminution in macular edema development after using a hypolipemiant agent (fibrates). These data are particularly important considering the extension of type 2 diabetes worldwide, and the fairly disappointing results of the treatment of diabetic retinopathy [44]. Fibrates (PPAR $\alpha$ agonist) could have anti-inflammatory and anti-oxidative/anti-apoptotic effects, and could furthermore improve vascular reactivity, thus attenuating the progression of diabetic retinopathy and the need for laser treatment. Further studies on oxidative stress and vascular inflammation in patients undergoing treatment with fibrates will define the mechanisms underlying microvascular benefits and could also prove to be useful in devising strategies for developing new drugs [44].

\section{The Role of PPARa Receptors in Rheumatoid Arthritis}

The hypothesis that PPAR $\alpha$ agonists (fibrates) could be used as anti-inflammatory agents, in treating both atherosclerosis, as well as patients with chronic inflammatory diseases (rheumatoid polyarthritis) -the fibrates inducing a decrease in TNF- $\alpha$, IL-1 $\beta$, IFN- $\gamma$ - has been proposed $[17,45]$. Experimental studies in vivo and in vitro have shown that PPAR $\alpha$ agonists inhibit bone resorption and reduce inflammation, the degradation of the synovial fluid, and the destruction of the articular cartilage. Consequently, PPAR $\alpha$ ligands reduce pain, diminish joint tumefaction and determine a decrease in inflammatory markers at systemic level [46].

\section{The "Antineoplastic" Effect of PPARa}

The role of PPAR $\alpha$ receptors in carcinogenesis is the subject of many discussions in the literature [5, 47, 48]. The administration of PPAR $\alpha$ agonists in the liver of animals has been observed to determine an increase in cell proliferation (through a PPAR $\alpha$-mediated mechanism) [49], while the same effect of stimulation of the appearance of tumor formations has not been observed in human patients undergoing treatment with fibrates for dyslipidemia (50). Thus, the existence of significant 
interspecies differences at the level of the liver between the effects of PPAR $\alpha$ stimulation in humans and animals has been suggested [50]. As regards colorectal cancer, PPAR $\alpha$ stimulation has been shown to inhibit the proliferation of tumor cell lines in the colon and to reduce the formation of adenomatous polyps on an animal experimental model of familial adenomatosis [51]. Moreover, in certain populations, an inverse association between the exposure to perfluorooctanoic acid (PPAR $\alpha$ agonist) and the prevalence of colorectal cancer has been reported [52]. The epidemiological studies have demonstrated the existence of a causal connection between chronic inflammation (occurring in the context of a microbial infection or of autoimmune diseases) and the appearance of tumor formation $[52,53]$. The role of PPAR $\alpha$ in carcinogenesis is the result of the inhibition of the nuclear factor NF-kB, ceasing the induction of the carcinogenic factors mentioned [54]. The antineoplastic action of PPAR $\alpha$ receptors is observed only in carcinogenesis with inflammatory determinism, absent in tumors in which inflammation is not involved.

\section{PPARa in Cutaneous Inflammation}

Nuclear receptors are transcription factors that control the activity of the enzymes involved in carbohydrate and lipid metabolism and are consequently involved in regulating energy and lipid homeostasis in the skin. PPAR $\alpha$ agonists control the proliferation/differentiation of keratinocytes and regulate the inflammation in the tegument [55], through the inhibiting action of the nuclear factor NFkB, followed by the inhibition of the TSLP (thymic stromal lymphopoietin) synthesis. Currently, TSLP protein is considered to be essential in the evolution of chronic diseases characterized by allergic inflammation, including atopic dermatitis, allergic rhinitis, and bronchial asthma [56]. The topical application of PPAR $\alpha$ agonists produces anti-inflammatory effects in skin disorders such as contact dermatitis, atopic dermatitis, solar erythema, consecutively improving the evolution of these disorders, irrespective of the application of topical steroids [57]. Moreover, alterations in genes involved in inflammation (MCP-1) and lipid metabolism (LXR $\alpha$, PPAR $\alpha$ ) have been observed in patients with psoriasis, thus identifying a connection between psoriasis and cardio-metabolic diseases. Consequently, an improvement in the prognosis of patients with psoriasis is expected after undergoing treatment with PPAR $\alpha$ antagonists [58].

\section{The Role of PPARa Receptors in Chronic Respiratory Diseases}

Allergic bronchial asthma is characterized by bronchial hyper reactivity, eosinophilia, being associated with elevated serum of IgE immunoglobulin. On animal models of bronchial asthma [59], PPAR $\alpha$ nuclear receptors have been shown to reduce bronchial hyper reactivity and the release of proinflammatory cytokines, simultaneously with the decrease in levels of circulating IgE. Moreover, the decrease of eosinophil chemotaxis in vitro and the inhibition of antigen-induced cell cytotoxicity have also been observed. PPAR $\alpha$ agonists could be said to constitute a new class of drugs in inflammatory or allergic reactions through the influence which they exert on the effector and regulatory cells involved in the body's immune response. The role of PPAR $\alpha$ has been studied in pulmonary fibrosis [60]. In the absence of the PPAR $\alpha$ receptor, experimental studies carried out on animals treated with bleomycin have highlighted an increased expression of TNF $\alpha$ and IL-1 $\beta$, an intensified apoptosis in interstitial cells, exacerbated inflammatory phenomena and pulmonary fibrosis and, consecutively, a decreased survival rate. In the same study, the treatment with PPAR $\alpha$ antagonists (WY-14643) has improved the survival rate, concomitant with the reduction of the TNF $\alpha$ cytokine and the bleomycin-induced fibrosis. It appears that the intervention mechanism of the PPAR $\alpha$ receptors in COPD is related to the inhibition of NF- $\kappa B$ and AP-1 pro-inflammatory factors [61]. 


\section{Conclusions}

The chronic diseases have been associated with a persistent, reduced level of pro-inflammatory factors. The discovery of insulin resistance and of type 2 diabetes is closely related to the infiltration of immune cells and chronic inflammation in the adipose tissue. In cardiovascular diseases, the activated immune cells and chronic inflammation play a major role, especially in the pathogenesis of atherosclerosis. Furthermore, tumor onset and progression is stimulated by the systemic increase in pro-inflammatory cytokines.

Atherosclerosis, perceived as a metabolic complication, draws attention to PPAR $\alpha$ receptors, transcription factors involved in lipid metabolism, inflammation and atheromatosis.

The action of PPAR $\alpha$ is manifested especially in energy-consuming tissues and organs, skeletal muscles, heart, liver. PPAR $\alpha$ activation determines a decrease in serum triglyceride and HDL-col levels and shields against an atherogenic lipid profile, by modulating the pro-inflammatory status and reducing insulin resistance.

\section{Conflicts of Interest}

The authors declare no conflicts of interest.

\section{References}

[1] A.S. Gami et al., Metabolic Syndrome and Risk of Incident Cardiovascular Events and Death, Journal of the American College of Cardiology. 49(5) (2007) 403-404.

[2] E. Cercosimo, R.A. Defronzo Insulin resistance and endothelial dysfunction: the road map to cardiovascular diseases, Diabetes Metab. Res. Rev. 22(6) (2006) 423-436.

[3] S. Azhar, Peroxisome proliferator-activated receptors, metabolic syndrome and cardiovascular disease, Future Cardiology. 6(5) (2010) 657-691.

[4] G. Andries, J.Ch. Fruchart, Peroxisome Proliferator-Activated Receptors at the Crossroads of Obesity, Diabetes and Cardiovascular Disease, Journal of American College Cardiology. 48(Supp19) (2006) 24-32.

[5] S. Kersten, B. Desvergne, W. Wahli, Roles of PPARS in health and disease, Nature. 405(6785) (2000) 421-424.

[6] A. Yessoufou, W. Wahli, Multifaceted roles of peroxisome proliferator-activated receptors (PPARs) at the cellular and whole organism levels, Swiss Med. Wkl. 140 (2010) w13071.

[7] S.J. Roberts-Thomson, Peroxisome proliferator-activated receptors in tumorigenesis: targets of tumour promotion and treatment, Immunology and Cell Biolog. 78(4) (2000) 36-41.

[8] D. Yusuf et al., The transcription factor encyclopedia, Genome Biol. 13(3) (2012) 1-30.

[9] J.K. Reddy et al., Chemical carcinogens without mutagenic activity: peroxisome proliferators as a prototype, Toxicol. Pathol. 11(2) (1983) 172-180.

[10] C.J. Pol, M. Lieu, K. Drosatos, PPARs: Protectors or Opponents of Myocardial Function?, PPAR Res. 2015 (2015) 835985.

[11] M.D. Li, X. Yang, A Retrospective on Nuclear Receptor Regulation of Inflammation: Lessons from GR and PPARs. PPAR Res 2011;2011:742785.

[12] J.P. Vanden Heuvel, J.M. Peters, Peroxisome Proliferators, in: K. Ramos (Ed.), Cellular and Molecular Toxicology (Second Edition), Vol/ 2, New York: Elsevier Science Inc., 2010, pp. 145-167. 
[13] M. Ricote, A.F. Valledor, C.K. Glass, Decoding transcriptional programs regulated by PPARs and LXRs in the macrophage: effects on lipid homeostasis, inflammation, and atherosclerosis, Arteriosclerosis, Thrombosis and Vascular Biology. 24(2) (2004) 230-239.

[14] E. Popa et al., Flow cytometry analysis of PPAR alpha receptors in metabolic syndrome. Romanian Journal of Laboratory Medicine. 22(4) (2014) 427-438.

[15] A. Zambon et al., Modulation of hepatic inflammatory risk markers of cardiovascular diseases by PPAR-alpha activators: clinical and experimental evidence, Arterioscler. Thromb. Vasc. Biol. 26(5) (2006) 977-986.

[16] J. Youssef, M. Badr, Role of Peroxisome Proliferator-Activated Receptors in Inflammation Control, Journal of Biomedicine and Biotechnology. 2004(3) (2004) 156-166.

[17] I.V. Shirinsky, V.S. Shirinsky Targeting Nuclear Hormone Receptors: PPAR $\alpha$ Agonists as Potential Disease-Modifying Drugs for Rheumatoid Arthritis, Int. J. Rheumatol. 2011 (2011) 937843.

[18] M. Rakhshandehroo et al., Peroxisome proliferator-activated receptor alpha target genes, PPAR Res. 2010 (2010) 612089.

[19] R. Belfort et al., Fenofibrate reduces systemic inflammation markers independent of its effects on lipid and glucose metabolism in patients with the metabolic syndrome, Journal of Clinical Endocrinology and Metabolism. 95(2) (2010) 829-836.

[20] D.S. Straus, C.K. Glass, Anti-inflammatory actions of PPAR ligands: new insights on cellular and molecular mechanisms, Trends Immunol. 28(12) (2007) 551-558.

[21] E. Cercosimo, R.A. Defronzo, Insulin resistance and endothelial dysfunction: the road map to cardiovascular diseases. Diabetes Metab. Res Rev. 22(6) (2006) 423-436.

[22] M.N. Sack et al., Fatty acid oxidation enzyme gene expression is downregulated in the failing heart, Circulation. 94(11) (1996) 2837-2842.

[23] B. Finck et al., A critical for PPAR $\alpha$-mediated lipotoxicity in the pathogenesis of diabetic cardiomyopathy: Modulation of phenotype by dietary fat content, Proc. Natl. Acad. Sci. 100(3) (2003) 1226-1231.

[24] S. Kersten Regulation of nutrient metabolism and inflammation, Results and Problems in Cell Differentiation. 52 (2010) 13-25.

[25] G.D. Lopaschuk et al., Myocardial fatty acid metabolism in health and disease, Physiological Reviews. 90(1) (2010) 207-258.

[26] C.L. Pol, M. Lieu, K. Drosatos, PPARs: Protectors or Opponents of Myocardial Function?, PPAR Research. 2015 (2015) Article ID 835985.

[27] S. Ares-Carrasco et al., Proteome changes in the myocardium of experimental chronic diabetes and hypertension: Role of PPAR $\alpha$ in the associated hypertrophy, Journal of Proteomics. 75(6) (2012) 1816-1829.

[28] S. Purushothaman, M.M. Sathik, R.R. Nair, Reactivation of peroxisome proliferator-activated receptor alpha in spontaneously hypertensive rat: age-associated paradoxical effect on the heart, Journal of Cardiovascular Pharmacology. 58(3) (2011) 254-262.

[29] P.M. Barger et al., Deactivation of peroxisome proliferator-activated receptor-alpha during cardiac hypertrophic growth, J. Clin. Invest. 105(12) (2000) 1723-1730.

[30] P.H. Lou et al., Infarct-remodelled hearts with limited oxidative capacity boost fatty acid oxidation after conditioning against ischaemia/reperfusion injury, Cardiovascular Research. 97(2) (2013) 251-261. 
[31] E.E. Morgan et al., Effects of chronic activation of peroxisome proliferator-activated receptoralpha or high-fat feeding in a rat infarct model of heart failure, The American Journal of Physiology-Heart and Circulatory Physiology. 290(5) (2006) H1899-H1904.

[32] W.S. Lee, J. Kim, Peroxisome, Proliferator-Activated Receptors and the Heart: Lessons from the Past and Future Directions, PPAR Research. 2015 (2015) Article ID 271983.

[33] B.N. Finck et al., A potential link between muscle peroxisome proliferator-activated receptor$\alpha$ signaling and obesity-related diabetes, Cell Metabolism. 1(2) (2005) 133-144.

[34] D.M. Muoio et al., Fatty acid homeostasis and induction of lipid regulatory genes in skeletal muscles of peroxisome proliferator-activated receptor (PPAR) $\alpha$ knock-out mice, Evidence for compensatory regulation by PPAR $\delta$, J. Biol. Chem. 277(29) (2002) 26089-26097.

[35] B.K. Pedersen, M.A. Febbraio, Muscles, exercise and obesity: skeletal muscle as a secretory organ, Nat. Rev. Endocrinol. 8(8) (2012) 457-465.

[36] A.G. Holmes et al., Prolonged interleukin-6 administration enhances glucose tolerance and increases skeletal muscle PPARalpha and UCP2 expression in rats, J. Endocrinol. 198(2) (2008) 367-374.

[37] F. Zandbergen, J. Plutzky PPAR $\alpha$ in atherosclerosis and inflammation, Biochim Biophys Acta. 1771(8) (2007) 972-982.

[38] C.F. Cheng, H.H. Chen, H. Lin, Role of PPAR $\alpha$ and Its Agonist in Renal Diseases, PPAR Resp 2010 (2010) 345098.

[39] S.M. De La Monte, J.R. Wands, Alzheimer's Disease Is Type 3 Diabetes-Evidence Reviewed, J. Diabetes Sci. Technol. 2(6) (2008) 1101-1113.

[40] N. Bhat, Linking cardiometabolic disorders to sporadic ad: a perspective on potential mechanisms and mediators, J. Neurochem. 115(3) (2010) 551-562.

[41] B. Staels et al., Mechanism of Action of Fibrates on Lipid and Lipoprotein Metabolism, Circulation. 98(19) (1998) 2088-2093.

[42] O. Kaidanovich-Beilin, D.S. Cha, R.S. McIntyre, Crosstalk between metabolic and neuropsychiatric disorders, F1000 Biol Reports. 4 (2012) 14. doi: 10.3410/B4-14.

[43] M.P. Hermans, Impact of Fenofibrate on Type 2 Diabetes Patients with Features of the Metabolic Syndrome: Subgroup Analysis From FIELD, Curr. Cardiol. Rev. 6(2) (2010) 112.

[44] A.C. Keech et al., Effect of fenofibrate on the need for laser treatment for diabetic retinopathy (FIELD study): a randomised controlled trial, The Lancet. 370(9600) (2007) 1687-1697.

[45] J. Lo Verme et al., Rapid broad-spectrum analgesia through activation of peroxisome proliferator-activated receptor-alpha, J. Pharmacol. Exp. Ther. 319(3) (2006) 1051-1061.

[46] I.C. Van Eekeren et al., Fibrates as therapy for osteoarthritis and rheumatoid arthritis? A systematic review, Ther. Adv. Musculoskelet Dis. 5(1) (2013) 33-44.

[47] M. Vacca et al., Clustering nuclear receptors in liver regeneration identifies candidate modulators of hepatocyte proliferation and hepatocarcinoma, PLoS One. 9(8) (2014) e104449.

[48] J. Youssef, M. Badr, Peroxisome proliferator-activated receptors and cancer: challenges and opportunities, Br. J. Pharmacol. 164(1) (2011) 68-82.

[49] Jo.A. Van Ginderachter, K. Movahedi, J. Van den Bossche, Macrophages, PPARs, and Cancer, PPAR Research. 2008 (2008) 169414. doi:10.1155/2008/169414.

[50] J.M. Peters, R.C. Cattley, F.G. Gonzalez, Mechanism of action of the nongenotoxic carcinogen and peroxisome proliferator Wy-14,643, Carcinogenesis. 18(11) (1997) 2029-2033. 
[51] M.W. Matthiessen et al., Peroxisome proliferator-activated receptor expression and activation in normal human colonic epithelial cells and tubular adenomas, Scand. J. Gastroenterol. 40(2) (2005) 198-205.

[52] K.E. Innes et al., Inverse association of colorectal cancer prevalence to serum levels of perfluorooctane sulfonate (PFOS) and perfluorooctanoate (PFOA) in a large Appalachian population, BMC Cancer. 14 (2014) 45. doi:10.1186/1471-2407-14-45.

[53] W.W. Lin, M. Karin, A cytokine-mediated link between innate immunity, inflammation, and cancer, J. Clin. Invest. 117(5) (2007) 1175-1183.

[54] T.W. Owens et al., Inhibitor of Apoptosis Proteins: Promising Targets for Cancer Therapy, J. Carcinog. Mutagen. Suppl. 14 (2013) S14-004.

[55] S. Dubrac, M. Schmuth, PPAR-alpha in cutaneous inflammation, Dermatoendocrinol. 3(1) (2011) 23-26.

[56] K. Verstraete et al., Structural basis of the proinflammatory signaling complex mediated by TSLP, Nat. Struct. Mol. Biol. 21(4) (2014) 375-382.

[57] M.Y. Sheu et al., Topical peroxisome proliferator activated receptor-alpha activators reduce inflammation in irritant and allergic contact dermatitis models, J. Invest. Dermatol. 118(1) (2002) 94-101.

[58] N.N. Mehta, et al., Modulation of cardiometabolic pathways in skin and serum from patients with psoriasis, J. Transl. Med. 11 (2013) 194. doi: 10.1186/1479-5876-11-194.

[59] C.D. Orthez et al., Suppression of allergen-induced airway inflammation and immune response by the peroxisome proliferator-activated receptor-alpha agonist fenofibrate, Eur. J. Pharmacology. 581(1-2) (2008) 177-184.

[60] M.G. Belvisi, J.A. Mitchell, Targeting PPAR receptors in the airway for the treatment of inflammatory lung disease, Br. J. Pharmacol. 158(4) (2009) 994-1003.

[61] E.M. Drost et al., Diminished peroxisome proliferator-activated receptor (PPAR) regulation as a potential mechanism for the persistent inflammation in chronic obstructive pulmonary disease, Eur. Resp. Rev. 15(101) (2006) 211-212. 